\title{
Multicast Broadcast Services Support in OFDMA-Based WiMAX Systems
}

\author{
Tao Jiang and Weidong Xiang, University of Michigan, Dearborn \\ Hsiao-Hwa Chen, National Cheng Kung University \\ Qiang Ni, Brunel University
}

\begin{abstract}
Multimedia stream service provided by broadband wireless networks has emerged as an important technology and has attracted much attention recently. An all-IP network architecture with reliable high-throughput air interface makes orthogonal frequency division multiplexing access (OFDMA)-based mobile worldwide interoperability for microwave access (mobile WiMAX) a viable technology for wireless multimedia services, such as voice over IP (VoIP), mobile TV, and so on. One of the main features in a WiMAX MAC layer is that it can provide differentiated services among different traffic categories with individual QoS requirements. In this article, we first give an overview of the key aspects of WiMAX and describe multimedia broadcast multicast service (MBMS) architecture of the 3GPP. Then, we propose a multicast and broadcast service (MBS) architecture for WiMAX that is based on MBMS. Moreover, we enhance the MBS architecture for mobile WiMAX to overcome the shortcoming of limited video broadcast performance over the baseline MBS model. We also give examples to demonstrate that the proposed architecture can support better mobility and offer higher power efficiency.
\end{abstract}

\section{INTRODUCTION}

Multimedia multicast and broadcast service (MBS) over wireless links, such as mobile TV and IP radio broadcasting, has become a fast growing application. As one of the most promising enabling technologies, mobile worldwide interoperability for microwave access (WiMAX) can offer scalability in both radio access and network architecture, thus providing important flexibility in terms of network services and deployment options [1, 2].

Mobile WiMAX, which is based on a nextgeneration all-IP core network, is a broadband wireless access solution that enables convergence of mobile and fixed broadband networks through a common wide area broadband radio access technology and flexible network architecture.
Moreover, mobile WiMAX can use multiple input multiple output (MIMO) with flexible subchannelization, adaptive coding, and modulation to support high data rate transmission.

Mobile WiMAX supports MBS combined with the features of digital video broadcast handheld (DVB-H) [3], media forward link only (MediaFLO) [4], and the third generation partnership project (3GPP) [5]. At the same time, mobile WiMAX air interface utilizes orthogonal frequency division multiplex (OFDM) or orthogonal frequency division multiple access (OFDMA) as the radio access scheme to improve system performance in non-line-of-sight (NLOS) communication environments $[1,2]$. Mobile WiMAX air interface can support [6]:

- High data rate and large coverage using a single frequency network (SFN)

- Flexible radio resources allocation that enables full or partial allocation of radio resources to MBS dynamically

- Low power consumption at the mobile station/subscriber station (MS/SS)

- Data-casting in addition to audio and video streams

- Low channel switching time to allow multichannel broadcast services such as mobile TV

- High macro-diversity gains in the SFN mode of operation due to the use of the OFDMA physical layer (PHY) and its properlydesigned frame structure

The WiMAX Forum has defined mechanisms for the initial MBS service content of mobile WiMAX delivery over the mobile WiMAX air interface. The MBS can be supported by either constructing a separate MBS zone in the downlink (DL) frame with unicast service, or the entire frame can be dedicated to MBS for broadcast service (DL only). The MBS zone supports the multi-BS MBS mode using a SFN operation, and flexible duration of MBS zones permits the scalable assignment of radio resources to MBS traffic. Note that multiple MBS zones are feasible. The multi-BS MBS does not require that the MS/SS be registered with a base station (BS). The MBS can be accessed when $\mathrm{MS} / \mathrm{SS}$ is in idle mode to reduce 
MS/SS power consumption. The flexibility of mobile WiMAX to support embedded MBS and unicast services enables a broad range of applications.

The rest of this article is organized as follows. We briefly review the WiMAX system based on OFDMA and introduce the MBMS architecture used in the 3 GPP. Then, we propose an MBS architecture according to the requirements for multimedia stream services in WiMAX systems. To overcome the problem with the baseline MBS model, which cannot offer a good video broadcast performance, we introduce an enhanced MBS architecture for mobile WiMAX, the effectiveness of which is demonstrated by examples, followed by the conclusion of the article.

\section{OFDMA-BASED WIMAX SYSTEM}

As specified in the standard, WiMAX employs OFDM technology in its PHY layer to support mesh and point-to-multi-point (PMP) networks. In fact, IEEE 802.16 has defined two types of OFDM systems: OFDM and OFDMA. OFDM is a multi-carrier modulation technique, whereas OFDMA is a multiple access scheme, in which data streams from multiple users are orthogonally multiplexed onto downlink (DL) and uplink (UL) subchannels/subcarriers.

OFDMA-based WiMAX system employs a larger fast Fourier transform (FFT) size (2048 and 4096 subcarriers) that is further divided into subchannels. The subchannels are introduced to separate the data into logical streams in DL. Those streams may employ different modulations, coding schemes, and amplitude levels to address subscribers with different channel characteristics. The subchannels also are used for multiple access in UL. In practice, the subscribers are assigned to subchannels through media access protocol (MAP) messages sent in downstream. OFDMA also enables smart antenna operations to be performed on vector-flat subcarriers based on smart antenna technologies that typically involve complex vector or matrix operations on signals due to the use of multiple antennas.

OFDMA-based WiMAX air interface supports both time division duplex (TDD) and frequency division duplex (FDD) modes in its PHY layer. For TDD mode, the system transmits the data frame-by-frame, and each 5-ms frame consists of a DL subframe and an UL subframe to prevent collisions between DL and UL transmissions. Adaptive modulation, fast channel feedback and link adaptation, coding, and asynchronous hybrid-automatic repeat request (H-ARQ) in DL and UL are used in the mobile WiMAX air interface to enhance its coverage and capacity. Different modulations (e.g., quadrature phase shift keying (QPSK)), channel coding schemes (i.e., convolution code (CC) and convolution turbo code (CTC)), space time block coding (STBC), and spatial multiplexing (SM) also are supported in mobile WiMAX TDD mode. Some of the scalability features of the PHY layer in TDD mode of mobile WiMAX are summarized in Table I [2].
According to the IEEE 802.16e standard [1], an MBS zone is defined as a specific region allocated by a BS or a group of BS in the DL frame along with MBS services. BS can send multicast/broadcast data synchronously through the same connection identifier (CID) and security association (SA) carrying MBS data in the same MBS zone. Users can obtain the information for an MBS zone from the received data transmitted from a BS. Note that one BS may belong to several MBS zones. Then, the MS and the SS can quickly confirm the MBS zone by DL-MAP. At the same time, there is an MBSMAP in an MBS zone, and MBS-MAP performs two functions:

- It indicates the location of the MBS packets.

- It informs the MS and SS when the next MBS packet is transmitted.

WiMAX is known to be connection-oriented, and thus, every connection between the MS/SS and the BS has its CID. For example, PMP transmission is implemented by multicast connection. Similarly, multicast CID (MCID) is required for MBS packets transmission.

To facilitate end-to-end interoperability and service environments, the WiMAX forum specified a WiMAX network reference model (NRM) that is a logical representation of the network architecture. An NRM is based on an all-IP core and packet-switched air interface. The main advantage of an NRM is that the network entities of the WiMAX system are agnostic to the IEEE 802.16 radio specifications, such as the IP multimedia subsystem and other protocols. Moreover, an NRM also allows the flexibility to configure logically related functional entities between various network entities.

Figure 1 illustrates the NRM [7] with the following logical entities: MS/SS, access service network (ASN), connectivity service network $(\mathrm{CSN})$, and the reference points for interconnection of the logical entities. Some key normative reference points, R1 and R6, also are shown in Fig. 1. MS/SS can be defined as the generalized collection of functions to provide connectivity between mobile/subscriber equipment and the BS. ASN is defined as a logical boundary that represents an aggregation of nodes in a mobile WiMAX radio access network. Typically, an ASN consists of multiple BS that perform radio-related functions and a gateway node (ASN-GW) that interfaces with a CSN - one of the key functional entities for WiMAX operators - and provides IP connectivity services to the WiMAX MS/SS.

Therefore, the end-to-end mobile WiMAX network architecture has the inherent capability to support micro-mobility and macro-mobility based handovers. The micro-mobility handovers typically are achieved between BS within the same ASN or adjacent ASN without changing the IP address. The macro-mobility handovers typically occur across ASN and may be accompanied with an IP address change. The WiMAX network architecture also supports inter-technology handovers, and it can support power efficient models of operation, such as idle mode and other advanced radio resource management schemes.
WiMAX is known to

be connection-

oriented, and thus,

every connection

between the MS/SS

and the BS has its

CID. For example,

PMP transmission is

implemented by

multicast connection.

Similarly, multicast

CID (MCID) is

required for $M B S$

packets transmission. 
MBMS is a

unidirectional PMP

service, in which

packets are

transmitted from a

single source entity

to multiple

endpoints. Therefore,

it is anticipated that

other services also

can use these

capabilities.

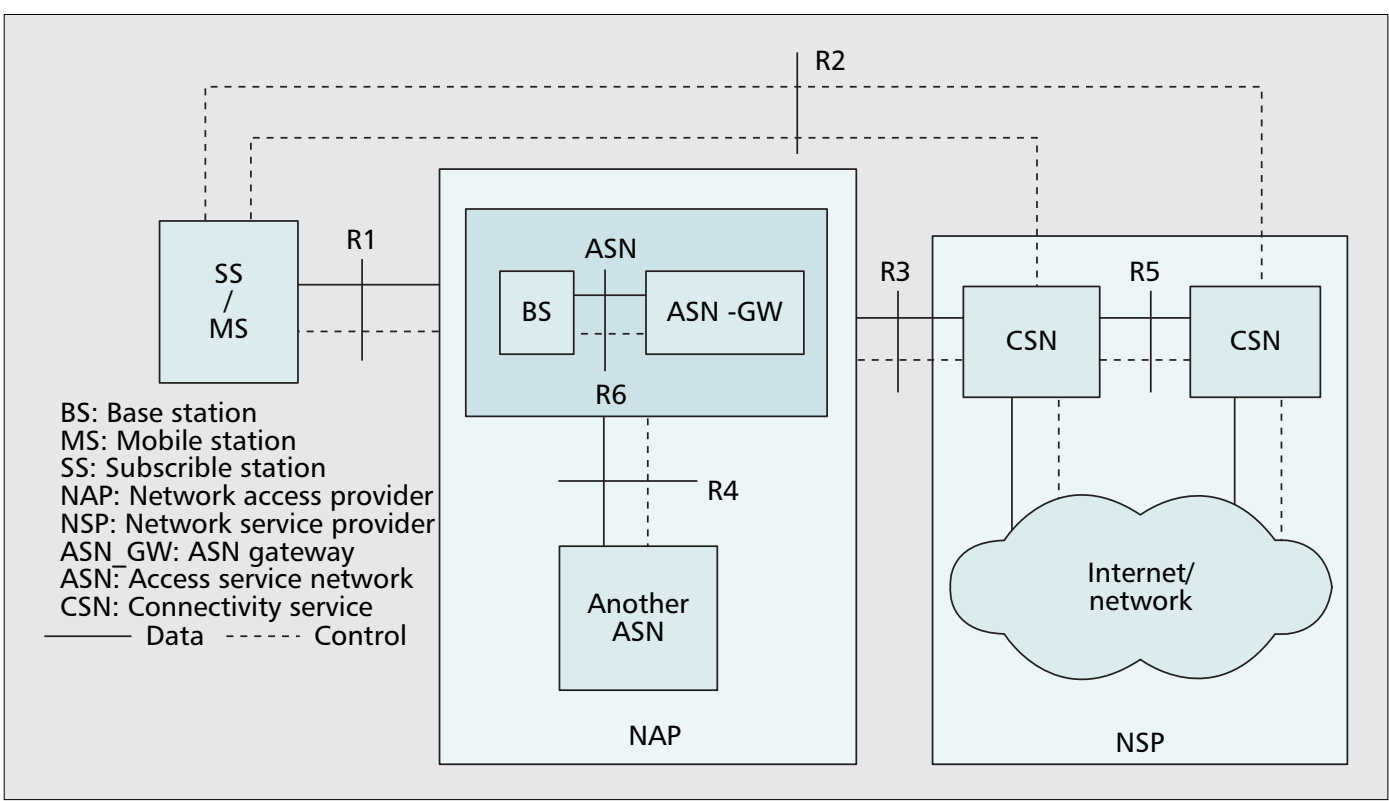

Figure 1. WiMAX network reference model (NRM).

\section{GPP MULTIMEDIA} BROAdCASt/MULTICAST SERVICES

To support the quality of service (QoS) required for multimedia stream services, $3 \mathrm{GPP}$ has standardized a general architecture: multimedia broadcast/multicast services (MBMS) [8]. MBMS is a unidirectional PMP service, in which packets are transmitted from a single source entity to multiple endpoints. Therefore, it is anticipated that other services also can use these capabilities. Moreover, the existing functional entities can be enhanced and a new functional entity, namely broadcast multicast service center (BMSC), also is added. BMSC provides a set of functions for the new MBMS user services including: membership, session and transmission, proxy and transport, service announcements, and security.

Just as its name implies, MBMS in 3 GPP bearer service offers two operational modes: broadcast network mode and multicast network mode, as shown in Fig. 2 [9]. A broadcast service must be able to distribute different content data to different locations, that is, local broadcast areas, within the broadcast service area as shown in Fig. 2. Broadcast network mode enables users to receive broadcast data depending on their locations, meaning that only one location-specific version of content data is distributed to each of the individual local broadcast areas. However, multicast service distributes different content data to different locations (local multicast areas) within the multicast service area, as shown in Fig. 2. Multicast network mode enables users to receive multicast data depending on their locations, meaning that only one version of locationspecific content data is distributed to each of the individual local multicast areas. Therefore, the main difference between these two network operation modes is that multicast mode has membership-related processes, such as joining and leaving processes, but broadcast mode does not.
There are two main contexts introduced to support MBMS in the 3GPP. One is the MBMS user equipment (UE) context, which contains UE-specific information related to a particular MBMS bearer service. The MBMS UE context can be created in the UE, the network controller (RNC), the serving-general packet radio service (GPRS) support node (SGSN), the gatewayGSN (GGSN), and the BMSC, whenever UE joins an MBMS bearer service. The other is the MBMS bearer context containing all information to describe a particular MBMS bearer service. Only when a downstream node, whose root is the BMSC in the distribution tree, requires the first MBMS UE context, the MBMS bearer context can be created in the radio RNC, SGSN, GGSN, and BMSC. Note that the MBMS UE context is mapped to each UE, whereas the MBMS bearer context is created for each multicast group that is represented by the distribution tree.

\section{Proposed MBS ARCHITECTURE FOR OFDMA-BASED WIMAX}

MBS is supported by both TDD and FDD models in the mobile WiMAX system to provide an efficient way to transmit diverse multimedia stream to multiple users through a shared radio resource [10]. The WiMAX standard defines two types of MBS: single-BS access and multi-BS access. Single-BS access is implemented within one BS, whereas multi-BS access transmits data over multiple BS to share multicast and broadcast connections. The multi-BS access uses SFN operation. In general, multiple BS compose an MBS zone in mobile WiMAX. Note that when all BS are in the same MBS zone, they have the same MCID for the same MBS multimedia stream transmission. Therefore, the multi-BS MBS does not require the MS to be registered to any BS. However, multi-BS access allows all MS to use the same multicast connection during 


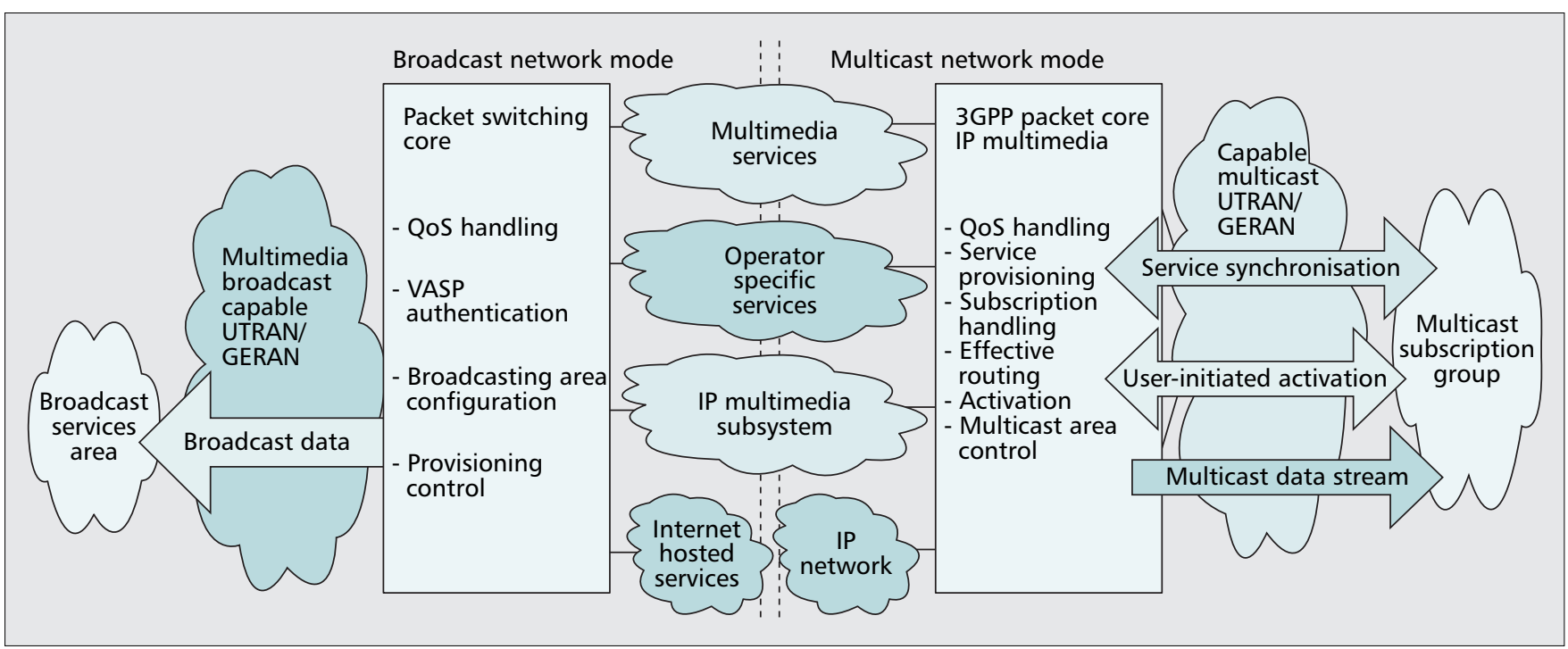

Figure 2. Broadcast and multicast network modes in 3GPP.

handovers within a single MBS zone, such that the MS can receive the MBS packets while moving within the MBS zone.

Since the initial mobile WiMAX profiles and releases are based on the TDD mode, we consider only the multi-BS operation in TDD mode for OFDMA-based mobile WiMAX in this article.

\section{NetWork ARChitecture REQUIREMENTS FOR MBS SUPPORT IN WIMAX}

The goal of the MBS for OFDMA-based mobile WiMAX is to incorporate the best features of DVBH, MediaFLO, and 3GPP MBMS. The support for MBS is a requirement for the future mobile WiMAX networks. Up to now, correlative architecture and protocols have been designed to meet the following requirements:

- Achieve maximum reuse of existing multicast and broadcast frameworks such as those defined in the $3 \mathrm{GPP}$ and $3 \mathrm{GPP} 2$ networks where they apply

- Optimize radio specific features and messages to take advantage of MBS capabilities defined in the medium access control (MAC) and PHY layers

- Comply and interwork with broadcast architecture at the application layer as defined by open mobile alliance

- Allow radio resource sharing between broadcast, multicast, and unicast transmissions

- Support multiple priority levels for MBS flows

- Enable flexible and dynamic allocation of the transmission zone and delivery mechanism

- Support various multimedia streams, such as still images and speech

- Enable effective service discovery/information acquisition, selection, and authorization
- Support QoS for real-time multimedia applications

- Enable multiple active MBS sessions and multiple active unicast sessions

- Support MBS delivery even in MS/SS power saving states, namely sleep and idle states

- Support application-level encryption of MBS content

- Allow content- and volume-based accounting and charging

- Collect MBS statistical data for operation, administration, maintenance, and provisioning usage

\section{PROPOSED MBS ARCHITECTURE FOR WIMAX}

Because WiMAX can be interworked with the 3GPP [11], we propose an MBS architecture for OFDMA-based mobile WiMAX, based on the MBMS architecture of the $3 \mathrm{GPP}$ according to the aforementioned requirements, which are shown in Fig. 3. We have added one additional functional entity into the WiMAX NRM, namely an MBS controller (MBSC), which is located in the CSN. MBSC is responsible for service delivery, service announcements, subscriber authentication, and so on. In the proposed architecture, the functions of each entity are described in detail as follows:

MS/SS: There are two application functions in the MS/SS, namely service discovery delivery and service subscription. The service discovery delivery function provides users with information on the forthcoming and ongoing MBS contents. Service subscription is associated with the subchannels.

ASN: According to the WiMAX standard [7], each ASN typically consists of one or more BS and a single ASN-GW. BS embodies WiMAX MAC and its PHY. The ASN-GW is a gateway IP router that supports IP multicast routing protocols. Because the WiMAX system is IP-based, users can join or leave via the Internet group management protocol (IGMP) that operates between MS/SS and the ASN-GW. 


MBSC performs
service requirements,
provisioning, and
delivery functions for
MBS services. It
serves as an entry
point for contents
providers, where
MBS transmission
services are
authorized and
initiated.

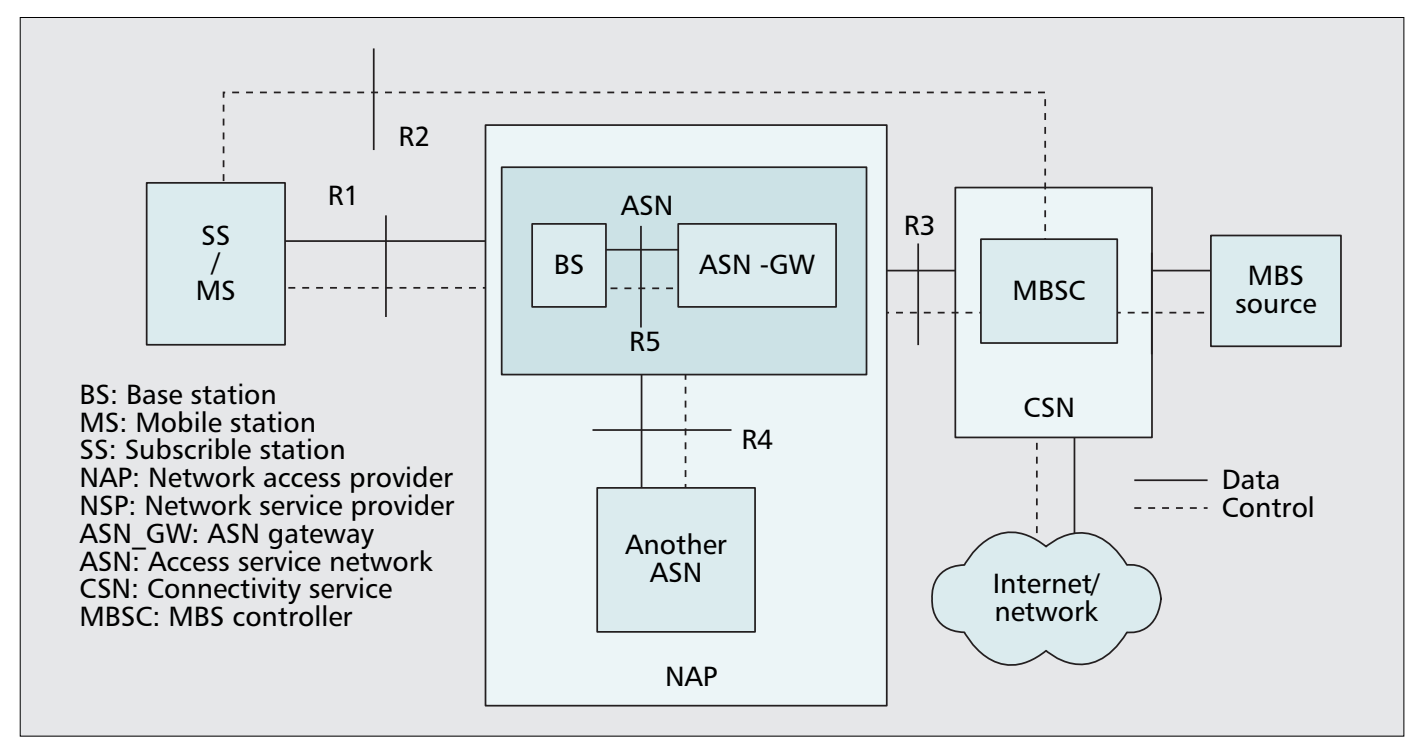

Figure 3. Proposed MBS architecture for mobile WiMAX.

MBS Source: This entity is used to offer applications, including real-time multimedia stream and non-real-time download for store and play usage, such as live sports events and weather reports. As the proposed WiMAX MBS architecture also is all IP-based, multimedia streams can be transmitted from any Internet terminal. The MBS architecture provides multimedia stream services to authorized MBS users based on subscription information.

MBSC: MBSC performs service requirements, provisioning, and delivery functions for MBS services. It serves as an entry point for contents providers, where MBS transmission services are authorized and initiated. The MBSC is a functional entity that performs six functions: service announcement, membership management, MBS zone management, MCID and IP address management, security management, and session management/transmission, as described in the following.

Service Announcements - This function provides potential MBS users with descriptions of the MBS channels and programs available. An MBS channel (including its programs), such as a news channel and a movie channel, is normally mapped to a single IEEE 802.16 multicast connection. The MBS channels and programs are distinguished by the logical channel ID and the MBS contents ID, respectively, which also are defined in the IEEE 802.16e [10]. Various service mechanisms (e.g., HTTP and push service) also can be used for the service announcement.

Membership Management - This function authorizes a user who requests to activate an MBS service. A subscription is normally associated with MBS channels or programs. This function must look up the subscription data of the MBS users.

MBS Zone Management - The MBSC delivers an MBS program to one or more MBS zones, each of which consists of multiple BS of a WiMAX network. MS/SS that access the same MBS channel in the same zone share a single
802.16 multicast connection. Note that all BS in the same MBS zone use the same key for encrypting the same MBS program. This reduces substantially the handoff delay between BS in the same MBS zone. Therefore, the MBSC must be aware of which BS constitute each MBS zone.

MCID and IP Address Management - This function performs mapping between an 802.16 MCID and an IP multicast address. The same IP multicast address is used between the ASNGW and the MS/SS by IGMP and also is used between the ASN-GW and MBSC by a multicast routing protocol (e.g., PIM-SM [10]). To correctly classify IP packets of MBS programs into 802.16 multicast connections, each BS requires the mapping information of the MCID and the IP multicast address when the MBSC creates a new MBS connection.

Security - This function provides key management, and thus, the IEEE 802.16 MAC privacy layer performs an integrity and confidentiality check of the MBS packets over the air interface. MS that access the same program in the same zone use the same cryptographic key for data decryption regardless of $\mathrm{BS}$. MBSC is responsible for creating, maintaining, and distributing a key for each MBS program in each zone.

Session Management/Transmission - An MBS session refers to a logical connection, established between an MS or SS and the MBSC, on which an MBS program is delivered to the users. An MS/SS identifies each session by the tuple of a channel identifier, namely logical channel ID, and an MBS content ID. The MBSC creates and maintains session information. It also schedules and transmits the packets of MBS sessions.

\section{ENHANCED MBS ARCHITECTURE FOR MOBILE WIMAX}

The IEEE 802.16e standard defined criteria only for the PHY and MAC layers. The basic model for the end-to-end multi-BS MBS is to use IP 


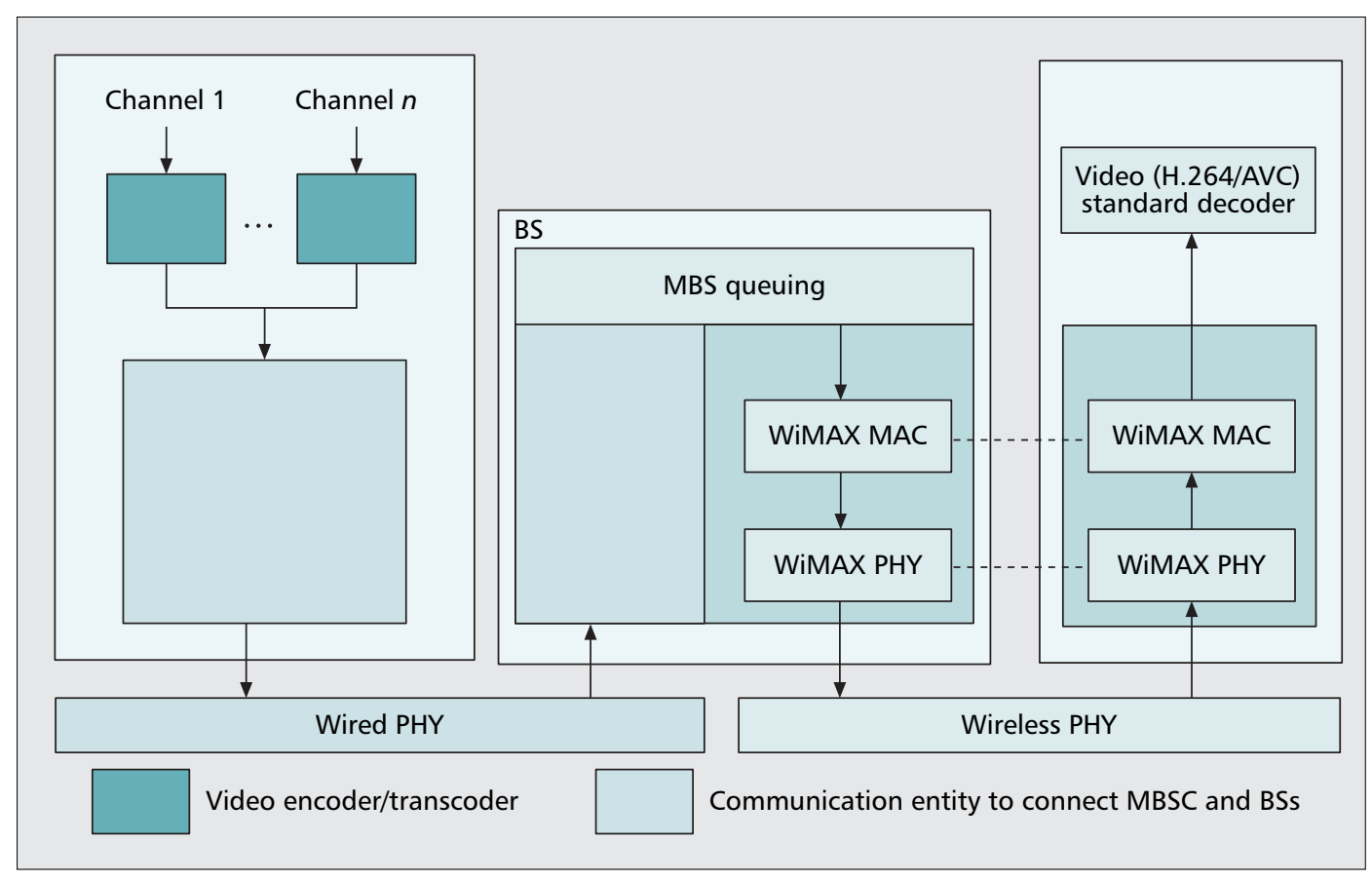

$$
\begin{aligned}
& \text { Due to variable bit } \\
& \text { rate (VBR) } \\
& \text { characteristics of } \\
& \text { video traffic, video } \\
& \text { packets might be } \\
& \text { required to be } \\
& \text { dropped randomly } \\
& \text { due to buffer } \\
& \text { overflow at the BS } \\
& \text { side, which could } \\
& \text { negatively impact } \\
& \text { video quality. }
\end{aligned}
$$

Figure 4. Baseline multi-BS MBS architecture for mobile WiMAX.

data cast of video over mobile WiMAX, as shown in Fig. 4. However, simple IP video data cast such as H.264/AVC have some issues as explained in the following.

- It is hard to achieve the synchronization of MBS across multi-BS. Synchronization means that the same video content is transmitted in the same TDD frame and in the same OFDMA data region using the same channel coding scheme. The MBS synchronization is critical not only for achieving macro diversity and reducing interference, but also for ensuring smooth handoff. The transmission delay of video data packets from the MBS controller to BS in the MBS zone varies over time and across BS. Video data packet duplicates also could be lost during transmission from the MBS controller to some BS. However, traditionally each BS makes its own scheduling decision. Therefore, the same OFDMA region will not transmit the same video data packet.

- Since the baseline scheme lacks error protection beyond PHY/MAC, large-size video frames could be error prone. To reduce frame errors, stronger channel coding schemes should be used in the PHY layer. However, channel coding leads to a lower spectral efficiency. In addition, if an unequal error protection scheme is not available, the video quality will degrade significantly when an MS/SS experiences shadow fading, temporal fading, or other interference. The idea of unequal error protection is to apply a more robust channel coding scheme to more important video content. Therefore, the MS/SS at least can decode some important video frames.

- Due to variable bit rate (VBR) characteristics of video traffic, video packets might be required to be dropped randomly due to buffer overflow at the BS side, which could negatively impact video quality.

- The energy efficiency could be low if a sim- ple IP video data-cast scheme does not use burst transmission, which is a mechanism to enable multiple MAC protocol data units (PDU) belonging to the same video channel to be transmitted/received in an aggregated way. Burst transmission enables MS/SS to save power by putting the transceiver in sleep mode during offburst interval. However, one must be cautious when using burst transmission. If the burst size is too large, the video channel switch delay will be too long. To save power, it is desirable for the MS/SS modem to decode only the received packets that correspond to the video channel currently being watched.

- The overhead of real-time transport protocol (RTP)/UDP/IP is significant. All of the aforementioned issues limit the video broadcast performance over a baseline MBS model.

Therefore, an enhanced MBS over mobile WiMAX must be proposed, as shown in Fig. 5. In mobile WiMAX PHY/MAC layers, there are three functional entities to support MBS over WiMAX: MBS server, MBS client, and the communication entity between MBS server and the BS.

The MBS server consists of a video encoder/transcoder and an MBS-enhanced transport-sublayer entity. The video encoder/transcoder conducts video encoding and RTP. The MBS-enhanced transport sub-layer entity is responsible for mapping the video channel identification to CID, shaping and multiplexing video traffic, encryption, performing Reed-Solomon outer coding, constructing the PDU fitting for transmission over WiMAX PHY/MAC, conducting burst scheduling, and allocating the OFDMA data region for each PDU.

The MBS client consists of a video channel switcher, a video decoder, and an MBS-enhanced transport sublayer entity. The mobile station 
Figure 5. Enhanced end-to-end MBS architecture for mobile WiMAX.

selects the video channel via a channel switcher that determines the MCID according to the selected video channel ID and indicates WiMAX PHY/MAC to decode only those PDU associated with the selected MCID. The MBS-enhanced transport sub-layer entity corrects errors of Reed-Solomon decoding sections, decrypts, and constructs the RTP video packet according to a standard video decoding algorithm.

The communication entity between the MBS server and the BS is provided for transporting video packets from the MBS server to multiple BS that are physically separated in the multi-BS MBS scenario. Note that, in the single-BS MBS scenario, the MBS server and BS may be integrated into one physical entity. In this case, the communication entity linking the MBS server with BS is no longer required.

If compared to the baseline system, the MBSenhanced transport sub-layer entity, the MBS queuing, and the mapping entity have been added in the proposed system. However, the enhanced MBS architecture can provide broadcast synchronization among multi-BS through the cooperation between the MBS server and BS and use Reed-Solomon outer coding and CTC inner coding to significantly reduce the video frame error rate without using too much overhead. Meanwhile, temporal scalability and unequal error protection for video streams can be provided to smooth the video quality degradation when users move within and across cells.

\section{Performance Analysis}

The performance of MBS over mobile WiMAX, which includes bandwidth efficiency, power saving, and mobility support, has been evaluated through analysis.

It is well-known that both the 3GPP MBMS and the WiMAX MBS architectures support PMP service not only over the air interface but also the infrastructure networks. Both architectures are efficient in terms of the usage of the network bandwidth. However, the maximum bit rate of a single MBS channel can be up to 2 $\mathrm{Mb} / \mathrm{s}$ in WiMAX [2], whereas the maximum bit rate of an MBMS channel is $384 \mathrm{~kb} / \mathrm{s}$ in the 3GPP [8]. Therefore, high quality multimedia contents can be serviced in broadband WiMAX.

Power efficient operation and handoff are the 
key aspects of mobility support in mobile WiMAX systems. The mobile WiMAX supports sleep and idle modes to enable power-saving MS operation. It also supports seamless handoff to enable the MS to switch from one BS to another at a vehicular speed without interrupting the connection. WiMAX MBS architecture can conserve energy by allowing an MS/SS to periodically awaken for the MBS service when MS/SS moves in the same MBS zone. Therefore, it is more efficient than that of the 3GPP MBMS model. The following scenario explains by using an example.

Suppose the bit rate is $360 \mathrm{Kpbs}$ and the spreading factor equals four in the 3GPP. Therefore, the MBMS packets in the 3GPP are delivered over a forward access channel $(\mathrm{FACH})$ and the data rate can be up to $360 \times 4=1440 \mathrm{~kb} / \mathrm{s}$. In $3 \mathrm{GPP}$, the UE should wake up and listen to FACH at least 25 percent of the duration, and it can sleep only when it does not receive MBMS packets and recalls. In practice, the UE of the MBMS service is always active during each MBMS session [5]. However, in mobile WiMAX operating in TDD mode with $10 \mathrm{MHz}$ channel, each frame has a 5-ms duration that consists of a preamble with one symbol, DL subframe with 26 symbols, and UL subframe with 15 symbols, as shown in Table 1. When modulation is QPSK and code rate is $1 / 2$, the data bits per single DL subframe in a typical subchannelization equal $1440 \times 26 \times 1 / 2=18720 \mathrm{bits} /$ frame, and 18720 bits/frame $\times 200$ frames $/$ second $=3744 \mathrm{~kb} / \mathrm{s}$ is supported by the BS. Therefore, the MS/SS in MBS mode is in sleep and idle modes up to (1 360/3744) 90.39 percent of the duration in DL subframes. In other words, MS/SS must be active only $360 / 3744 \times 26 /(1+26+15) \approx 5.95$ percent of the whole duration during each MBS session.

In addition, we have obtained simulation results of the coverage quality for a mobile WiMAX network. It has been shown that the coverage performance depends on the system parameters, including transmission power, cell size, and duration of the cyclic prefix. When 19 cells operate with the parameters of $2500 \mathrm{MHz}$ carrier frequency, $1 / 8$ cyclic prefix, and $20 \mathrm{~W}$ of the maximum power at BS, together with other corresponding parameters as listed in Table I, the mobile WiMAX network can provide about 95.5 percent coverage with a packet error rate lower than one percent in the center cell over International Telecommunication Union (ITU) Ped B channel.

\section{CONCLUSION}

In this article, we propose an architecture to support MBS service for a WiMAX system that is based on MBMS architecture in a 3GPP system. Moreover, an effort was made to enhance MBS architecture for OFDMA-based mobile WiMAX to overcome the shortcoming of degraded video broadcast performance over the baseline MBS model. The versatility of mobile WiMAX radio access network (RAN) and all-IP network architecture combined with high throughput, spectral efficiency, and data rates (if compared with 3 GPP systems) have made mobile WiMAX one of the most viable radio access

\begin{tabular}{|c|c|c|c|c|}
\hline Bandwidth (MHz) & 1.25 & 5 & 10 & 20 \\
\hline FFT size & 128 & 512 & 1024 & 2048 \\
\hline Frame size & \multicolumn{4}{|l|}{$5 \mathrm{~ms}$} \\
\hline Subcarrier frequency offset & \multicolumn{4}{|c|}{$10.94 \mathrm{kHz}$} \\
\hline Useful symbol time & \multicolumn{4}{|c|}{$91.4 \mu \mathrm{s}$} \\
\hline Cyclic prefix & \multicolumn{4}{|c|}{$11.4 \mu \mathrm{s}$} \\
\hline OFDM symbol duration & \multicolumn{4}{|c|}{$102.9 \mu \mathrm{s}$} \\
\hline Numbers of OFDM symbols per frame & \multicolumn{4}{|l|}{48} \\
\hline Data OFDM symbols & \multicolumn{4}{|l|}{44} \\
\hline Modulation for DL & \multicolumn{4}{|c|}{ QPSK/16-QAM/64-QAM } \\
\hline Modulation for UL & \multicolumn{4}{|c|}{ QPSK/16-QAM } \\
\hline Channel code & \multicolumn{4}{|c|}{$\mathrm{CC}, \mathrm{CTC}$} \\
\hline Coding rate of $\mathrm{DL}$ & \multicolumn{4}{|c|}{$1 / 2,2 / 3,3 / 4,5 / 6$} \\
\hline Coding rate of UL & \multicolumn{4}{|c|}{$1 / 2,3 / 4$} \\
\hline Repetition & \multicolumn{4}{|c|}{$\times 2, \times 4, \times 6$} \\
\hline MIMO and transmit diversity & \multicolumn{4}{|c|}{ STBC, SM } \\
\hline Downlink peak data rate & \multicolumn{4}{|c|}{$\begin{array}{l}46 \mathrm{Mb} / \mathrm{s} \text { when the ratio of } \mathrm{DL} \text { to } \mathrm{UL} \text { is } 3 \\
32 \mathrm{Mb} / \mathrm{s} \text { when the ratio of } \mathrm{DL} \text { to } \mathrm{UL} \text { is } 1 \\
\text { (10 MHz bandwidth, } 2 \times 2 \mathrm{MIMO})\end{array}$} \\
\hline Uplink peak data rate & \multicolumn{4}{|c|}{$\begin{array}{l}4 \mathrm{Mb} / \mathrm{s} \text { when the ratio of } \mathrm{DL} \text { to } \mathrm{UL} \text { is } 3 \\
7 \mathrm{Mb} / \mathrm{s} \text { when the ratio of } \mathrm{DL} \text { to } \mathrm{UL} \text { is } 1 \\
(10 \mathrm{MHz} \text { bandwidth, collaborative } \\
\text { MIMO) }\end{array}$} \\
\hline
\end{tabular}

Table 1. OFDMA scalability parameters of mobile WIMAX TDD model.

technologies to deliver a variety of unicast, broadcast, and multicast services and applications, including voice over IP (VoIP) and mobile TV. Through examples, we conclude that the proposed MBS architecture can support mobile services and offer more efficient power than that of the MBMS architecture adopted in 3GPP systems.

\section{REFERENCES}

[1] IEEE Std. 802.16e-2005, “Local and Metropolitan Area Networks Part 16: Air Interface for Fixed and Mobile Broadband Wireless Access Systems Amendment for Physical and Medium Access Control Layers for Combined Fixed and Mobile Operation in Licensed Bands," Dec. 2005.

[2] WiMAX Forum, "Mobile WiMAX - Part I: A Technical Overview and Performance Evaluation," June 2006; http://www.wimaxforum.org/

[3] M. Kornfeld, "DVB-H - The Emerging Standard for Mobile Data Communication," IEEE Int'l. Symp. Consumer Elect., vol. 1, Sept. 2004, pp. 193-98.

[4] QUALCOMM MediaFLO; http://www.qualcomm.com/ mediaflo

[5] 3GPP Tech. Spec. TS 25.346, "Introduction of the Multimedia Broadcast/Multicast Service (MBMS) in the Radio Access Network (RAN)," Mar. 2006; http://www.3gpp.org/ 
[6] WiMAX Forum, "Mobile WiMAX - Part II: A Comparative Analysis," May 2006; http://www.wimaxforum.org/

[7] WiMAX Forum, "WiMAX End-to-End Network Systems Architecture, Stage 2: Architecture Tenets, Reference Model and Reference Points," Aug. 2006; http://www. wimaxforum.org/

[8] 3GPP Tech. Spec. TS 23.246, "Multimedia Broadcast/ Multicast Service; Architecture and Functional Description (Release 6)" v. 6.9.0, Dec. 2005.

[9] 3GPP Tech. Spec. TS 22.246, "Multimedia Broadcast/Multicast Service (MBMS) User Services; Stage 1 (Release 7)," v. 7.0.0, Sept. 2005.

[10] IEEE Stds. 802.16e-2005 and 802.16-2004/Cor 1-2005, "Local and Metropolitan Area Networks Part 16: Air Interface for Fixed Broadband Wireless Access Systems Amendment for Physical and Medium Access Control Layers for Combined Fixed and Mobile Operation in Licensed Bands" and Corrigendum 1, 2004.

[11] WiMAX Forum, "WiMAX End-to-End Network Systems Architecture, Stage 3: WiMAX - 3GPP Interworking," Aug. 2006; http://www.wimaxforum.org/

\section{BIOGRAPHIES}

TAO JIANG [M'06] (Tao.Jiang@ieee.org) received his B.S. and M.S. degrees in applied geophysics from China University of Geosciences, Wuhan, in 1997 and 2000, respectively, and a Ph.D. degree in information and communication engineering from Huazhong University of Science and Technology, Wuhan, P. R. China, in April 2004. He is now a postdoctoral researcher in the Department of Electronic and Computer Engineering, University of Michigan, Dearborn. His current research interests include the areas of wireless communications and corresponding signal processing, especially for OFDM, UWB, and MIMO systems; cooperative networks; and cognitive radio. He is a member of the IEEE Communications and Broadcasting Societies.

WEIDONG XIANG (xwd@umich.edu) received his M.S. and Ph.D. degrees from Tsinghua University, Beijing, China, in 1996 and 1999, respectively. In September 2004 he joined the Department of Electrical and Computer Engineering, University of Michigan, Dearborn, as an assistant professor. His research interests include vehicular networks, software radio and cognitive radio, ultra wideband, and wireless networked control systems. From 1999 to 2004 he worked as a postdoctoral fellow and then as a research scientist in the Software Radio Laboratory at Georgia Institute of Technology, Atlanta.

HSIAO-HWA CHEN [SM'00] (hshwchen@ieee.org) received his B.Sc. and M.Sc. degrees with highest honors from Zhejiang University, China, and a Ph.D. degree from the University of Oulu, Finland, in 1982, 1985, and 1990, respectively, all in electrical engineering. In 2001 he joined National Sun YatSen University, Taiwan, as the founding director of the Institute of Communications Engineering of the university. Under his leadership, the institute was ranked second in the country in terms of $\mathrm{SCl}$ journal publications and National Science Council funding per faculty member in 2004. In particular, National Sun Yat-Sen University was ranked first in the world in terms of the number of SCl journal publications in wireless LAN research papers during 2004 to mid-
2005, according to a research report available at http://www.onr.navy.mil/sci tech/special/354/technowatch/te xtmine.asp and released by the Office of Navel Research in the United States. His current research interests include wireless networking, MIMO systems, next-generation CDMA technologies, information security, and beyond $3 \mathrm{G}$ wireless communications. He worked with the Academy of Finland as a research associate from 1991 to 1993 and the Nationa University of Singapore as a lecturer and then senior lecturer from 1992 to 1997. He joined the Department of Electrical Engineering, National Chung Hsing University, Taiwan, as an associate professor in 1997 and was promoted to a full professor in 2000. He was a visiting professor at the Department of Electrical Engineering, University of Kaiserslautern, Germany, in 1999, the Institute of Applied Physics, Tsukuba University, Japan, in 2000, Institute of Experimental Mathematics, University of Essen, Germany, in 2002 (under a DFG Fellowship), the Chinese University of Hong Kong in 2004, and the City University of Hong Kong in 2007. He is a recipient of numerous research and teaching awards from the National Science Council, the Ministry of Education, and other professional groups in Taiwan. He has authored or co-authored over 200 technical papers in major international journals and conferences, five books, and several book chapters in the areas of communications, including two books titled Next Generation Wireless Systems and Networks and The Next Generation CDMA Technologies (Wiley, 2005 and 2007). He has been an active volunteer in various IEEE technical activities for over 15 years. Currently, he is serving as Chair of the IEEE Communications Society Radio Communications Committee. He served or is serving as symposium chair/co-chair of many major IEEE conferences, including IEEE VTC '03 Fall, IEEE ICC '04, IEEE GLOBECOM '04, IEEE ICC '05, IEEE GLOBECOM '05, IEEE ICC '06, IEEE GLOBECOM '06, IEEE ICC '07, and IEEE WCNC '07. He served or is serving as an editorial board member and/or a guest editor of IEEE Communications Letters, IEEE Communications Magazine, IEEE Wireless Communications, IEEE JSAC, IEEE Network, IEEE Transactions on Wireless Communications, and IEEE Vehicular Technology Magazine. He is serving as Chief Editor (Asia and Pacific) for Wiley's Wireless Communications and Mobile Computing Journal and Wiley's International Journal of Communication Systems. His original work in CDMA wireless networks, digital communications, and radar systems has resulted in five U.S. patents, two Finnish patents, three Taiwanese patents, and two Chinese patents, some of which have been licensed to industry for commercial applications. He also is an adjunct Professor at Zhejiang University, China, and Shanghai Jiao Tung University, China.

QIANG Nı [M'04] (Qiang.Ni@brunel.ac.uk) is a faculty member of the School of Engineering and Design, Brunel University, West London, United Kingdom, where he heads the Intelligent Wireless Communication Networking Group. Prior to that he was with the Hamilton Institute, National University of Ireland Maynooth (2004-2005) and Planete Group INRIA France (2001-2004), respectively. His research interests are intelligent wireless networking and mobile communications including WLAN, WiMAX, wireless PAN, and 4G mobile networks. He received his Ph.D. degree in 1999 from Huazhong University of Science and Technology, China. 